\title{
Isolation of Multidrug Resistant Bacteria from Aspirates of Cancer Patients
}

\author{
Sunjukta Ahsan ${ }^{*}$, Rayhan Mahmud², Kajal Ahsan³ and Shamima Begum² \\ ${ }^{I}$ Department of Microbiology, University of Dhaka, Dhaka 1000, Bangladesh, ${ }^{2}$ Department of Microbiology, Jagannath University, Dhaka, Bangladesh, \\ ${ }^{3}$ Department of Cytopathology, National Institute of Cancer Research and Hospital, Mohakhali, Dhaka 1212, Bangladesh
}

\begin{abstract}
Infections due to Gram-negative bacteria are common affairs in cancer patients during aggressive therapy. The present study characterizedmulti-drug resistant bacteria (MDR) isolated from cancer aspirates collected from patients admitted to the National Cancer Hospital in Dhaka, Bangladesh. A total of 210aspirate samples were collected from cancer patients. Out of 210 samples Acinetobacter spp.led the list of isolates $(8.89 \%, n=45)$. Of these species, $50 \%$ exhibited resistance to Amoxycillin and Nitrofurantoin, each, 25\% exhibited resistant to Cefotaxime, Azithromycin, Ciprofloxacin, Clindamycin, and Sulfamethoxazole. A total of $33.33 \%$ of the Bordetella spp.which accounted $6.67 \%$ of the total isolates exhibited resistance to Cefotaxime. All oftheLegionellapneumophila,comprising 4.4\%of the isolated species, wereresistant to Cefotaxime, Azithromycin, and Clindamycin.In contrast, $50 \%$ were resistant to Cefotaxime, Azithromycin, and Ceftriaxone. Of the Escherichia coli $(4.4 \%, n=45)$ isolated,50\% exhibited resistance to Cefotaxime, Clindamycin, Ceftriaxone, Amoxycillinand Sulfamethoxazole.The only isolate of Klebsiella sp. was demonstrated to be an ESBL producer. The isolation of multidrug resistant bacteria from cancer patients is of particular concern in Bangladesh where cancer and drug resistance are both common phenomena but treatment facilities are poor. To our knowledge this is the first report of the isolation of drug resistant bacteria from cancer patients from Dhaka city.
\end{abstract}

\section{Introduction}

Multidrug resistance is a common affair among the people of third world. The earliest mechanisms by which many cancers develop resistance to chemotherapy drugs is a prime factor in the dysfunction of many forms of chemotherapy. It affects patients with a variety of blood cancers and solid tumors, including breast, ovarian, lung, and lower gastrointestinal tract cancers. Most patients with head and neck cancer have dysphagia and are at great risk of having aspiration and subsequent pneumonia. It can cause prolonged hospitalization, treatment delay and/or interruption and mortality in cancer patients. The treatment of these infections often relies on empirical antibiotics based on local microbiology and antibiotic sensitivity patterns ${ }^{1-2}$.

Multidrug resistance, the principal mechanism by which many cancers develop resistance to chemotherapy drugs, is a major factor in the failure of many forms of chemotherapy. Tumors usually consist of mixed populations of malignant cells, some of which are drug-sensitive while others are drug-resistant. Chemotherapy kills drug-sensitive cells, but leaves behind a higher proportion of drug-resistant cells. As the tumor begins to grow again, chemotherapy may fail because the remaining tumor cells are now resistant. Resistance to therapy has been correlated to the presence of at least two molecular "pumps" in tumor-cell membranes that actively expel chemotherapy drugs from the interior. This allows tumor cells to avoid the toxic effects of the drug or molecular processes within the nucleus or the cytoplasm.
The two pumps commonly found to confer chemo resistance in cancer are P-glycoprotein and the so-called multidrug resistanceassociated protein (MRP). Because of their function and importance, they are the targets of several anticancer efforts ${ }^{3}$.

In recent years, the emergence of antimicrobial resistance has become a significant problem worldwide, and cancer patients are among those affected with various species of both Gram positive and Gram negative bacteria. Treatment of infections due to multidrug-resistant (MDR) bacteria represents a clinical challenge, since the therapeutic options are often very limited. As the antibiotics active against MDR bacteria present several disadvantages (limited clinical experience, higher incidence of adverse effects, and less knowledge of the pharmacokinetics of the drug), a thorough acquaintance with the main characteristics of these drugs is mandatory in order to provide safe treatment to cancer patients with MDR bacterial infections ${ }^{4-5}$.The aims of the present study were to determine multi drug resistance of bacteria isolated from cancer patientsundergoing treatment at National Cancer Institute, Mohakhali, Dhaka, Bangladesh.

\section{Materials and methods}

Sample collection and processing

A total of 210 blood and aspirate samples were aseptically collected by a laboratory professional from various cancer patients after taking their consent. Samples were inoculated in TSB.Samples were transported to the laboratory in ice box and 
then incubated for 24 to 48 hour at $37^{\circ} \mathrm{C}$. The incubated broth was carefully transferred to a separate autoclaved clean Eppendorf tube, leaving behind the blood pellet and centrifuged for $3 \mathrm{~min}$ at $10,000 \mathrm{rpm}$ to collect all the cells as growth was seen to be verypoor. This centrifugation process was done to concentrate the cell before inoculate in the culture media.Incubated culture samples were stored by adding $95 \%$ glycerol at $-20^{\circ} \mathrm{C}$ for further research.

\section{Bacteriological analysis of the clinical samples}

After 24 to 48 hours incubation at $37^{\circ} \mathrm{C}$, on the basis of visual turbidity centrifuged culture samples $100 \mu 1$ were inoculated in nutrient agar plates by spread plate technique. If the culture appeared to be too turbid then streak plate method was applied. All the plates were incubated for 24 to 48 hours at $37^{\circ} \mathrm{C}$ to have the isolates. After incubation primary plates were screened to identify different colonies for Gram staining and subculture.

\section{Storage of bacterial isolates}

For preservation, $300 \mu \mathrm{l}$ of fresh bacterial culture grown in TSB at $37^{\circ} \mathrm{C}$ for 24 to $48 \mathrm{~h}$ was taken in a sterile Eppendorf tube and $700 \mu \mathrm{l}$ of sterile glycerol (95\%)was added to the broth culture; culture broth and glycerol was mixed by vortexing. The Eppendorf tube was stored at $-20 \mathrm{C}$ with proper labeling.

\section{Morphological characteristics}

Colony morphology is a method that scientists use to describe the characteristics of an individual colony of bacteria growing on agar in a Petri dish. It can be used to identify them. Different types of bacteria produce differentcolony characteristics. Colony characteristics were observed following growth in Nutrient agar and MacConkey agar.

\section{Gram staining}

A small portion of bacterial fresh culture was taken out with a sterile inoculating loop and was placed on previously cleaned oil free slide. A thin smear was prepared and allowed to dry in air. The smear was heat fixed by slightly heating under surface of a slide over a gas burner. Crystal violet, the primary stain, was flooded and left over the smear for one minute and readily washed with slow moving tap water. The smear was then flooded with Gram's iodine as mordant to stabilize the color of the primary stain. Allowing one minute the smear was washed again with slow moving tap water thoroughly. The smear was then decolorized by the applying $95 \%$ ethanol or acetone for 5 to 10 seconds and raised well with tap water. Finally, the secondary stain Safranin was applied for one minute and subsequently washed off with distilled water. The slide was then kept for air-drying before microscopy. The dried smear was then examined under oil-immersion lens.

\section{Biochemical characterization}

Biochemical tests viz. catalase, oxidase, Indole, Methyl red, Voges-Proskauer test, Motility Indole Urease test, Nitrate Reduction test, Citrate utilization, Lysine Decarboxylase test, Coagulase test, Kligler Iron Agar test,

\section{Biochemical identification through software}

Identification of species was done by the aid of an online software, Gideon ${ }^{6}$.

\section{Antimicrobial susceptibility test}

Antimicrobial susceptibility test was carried out by the standard disc diffusion test described previously ${ }^{7}$. A total of ten antibiotics were used to identify the antibiogram of the bacteria. These were CTX - Cefotaxime, AZM - Azithromycin, AMC - Amoxycillin, DA - Clindamycin, SXT - Sulfamethoxazole, CRO - Ceftriaxone, AML - Amoxyclav, CIP - Ciprofloxacin, TE - Tetracycline, and $\mathrm{F}-$ Nitrofurantoin. Isolates that exhibited resistance to the ${ }^{2}$ lactam antibiotics were tested for ESBL production by using the double disk diffusion test as describedpreviously ${ }^{8}$. After overnight incubation at $37^{\circ} \mathrm{C}$, any enhancement of the zone of inhibitionbetween a beta-lactam disk and that containing the betalactamaseinhibitor was indicative of the presence of an ESBL.

\section{Results}

\section{Bacteria isolation}

A total of 210 samples were studied. Of the samples, $53.8 \%$ were from female patients and $46.2 \%$ from males. The sites of cancer in these patients were $0.48 \%$ in ovary and lymph node each, $0.95 \%$ in kidney, $1.42 \%$ in breast and liver each and $95.24 \%$ in blood. The isolates were primarily identified on the basis of their colony morphology and cultural characteristics on the MacConkey agar (MAC), Eosin Methylene Blue (EMB) and XLD agar plates. Gram Staining was performed. The percentages of male and female cancer patients investigated together with their age group is shown in Figure 1. The culture based characteristics and Gram staining reaction of different isolates are given in Table 1.

\section{Biochemical Tests for Identification of Isolates}

After growth on MacConkey and Eosine Methylene Blue Agar plates, the isolates were further identified by biochemical tests as Triple Sugar Iron (TSI) test, Motility Indole Urea (MIU) test, IMVIC test, MIO, Ornithine decarboxylase, Lysine decaboxylase, Catalase and Oxidase tests.An online software, Gideon ${ }^{6}$, was used for identification of the isolates. The frequencies of bacteria identified by Gideon are given in Figure 2.

\section{Antibiotic susceptibility of the isolates}

Following the Clinical and Laboratory Standard Institute(CLSI) guideline, most of the isolates were resistant to Cefotaxime, Nitrofurantoin,Clindamycin, Ceftriaxone, and Amoxycillin. Most of the isolates were sensitive to Ciprofloxacin (Figure 3).

\section{Extended spectrum ${ }^{2}$ lactamase (ESBL) activity of the isolates}

In this study 45 isolates $(55.6 \%, \mathrm{n}=45)$ were tested for ESBL production and only one isolate $(2 \%, \mathrm{n}=45)$ wasan $\mathrm{ESBL}$ producer. 
Table 1. The morphological characteristic of bacterial colonies on different media in the supplied sample.

\begin{tabular}{|c|c|c|c|c|c|}
\hline \multirow[t]{2}{*}{ Sample No. } & \multirow[t]{2}{*}{ MAC } & \multirow[t]{2}{*}{ EMB } & \multirow[t]{2}{*}{ XLD } & \multicolumn{2}{|c|}{ Gram Staining } \\
\hline & & & & Shape & $\mathrm{Ve}+/ \mathrm{ve}-$ \\
\hline$\overline{38 / \mathrm{s}}$ & White/gummy & - & - & rod & - \\
\hline 17 & White/gummy & - & + & coccus & - \\
\hline 24 & White/gummy & - & + & rod & - \\
\hline $52 / \mathrm{s}$ & Yellow/gummy & + & + & cocci & - \\
\hline 43 & White/gummy & - & - & $\operatorname{rod}$ & - \\
\hline 41 & White/gummy & - & - & cocci & - \\
\hline $58 / \mathrm{L}$ & Slightly yellow/gummy & + & + & rod & - \\
\hline $16 / \mathrm{C}$ & White/gummy & + & - & cocci & - \\
\hline 182 & Water like & - & - & $\operatorname{rod}$ & + \\
\hline 167 & White/gummy & + & - & rod & - \\
\hline 179 & Red/gummy & + & - & rod & - \\
\hline 162 & White/gummy & - & + & $\operatorname{rod}$ & - \\
\hline 149 & Water like & + & - & rod & - \\
\hline 32 & White/gummy & + & - & cocci & - \\
\hline 60 & Colorless & - & - & cocci & - \\
\hline 57 & pink/gummy & - & + & rod & - \\
\hline $16 \mathrm{w} / \mathrm{c}$ & White/gummy & + & + & $\operatorname{rod}$ & - \\
\hline 36 & Red/gummy & + & + & rod & - \\
\hline 40 & Pink/gummy & - & + & $\operatorname{rod}$ & - \\
\hline 181 & Pink/gummy & + & - & cocci & - \\
\hline 64 & Colorless & - & - & cocci & - \\
\hline 96 & Pink/gummy & + & - & rod & - \\
\hline 109 & Slightly red/huge gummy & + & - & rod & - \\
\hline 14 & Colorless & + & - & cocci & - \\
\hline 39 & Colorless & + & - & cocci & - \\
\hline 81 & Pink/gummy & + & + & $\operatorname{rod}$ & - \\
\hline $34 / \mathrm{s}$ & White/gummy & - & + & rod & - \\
\hline 207 & Colorless & - & - & cocci & - \\
\hline 30 & Colorless & + & - & cocci & - \\
\hline 94 & Colorless/gummy & - & + & cocci & - \\
\hline $36 / \mathrm{L}$ & Colorless/gummy & + & - & cocci & - \\
\hline 19 & Colorless/gummy & - & - & rod & - \\
\hline 12 & Pink/gummy & + & + & rod & - \\
\hline 18 & Colorless/gummy & - & - & rod & - \\
\hline 20 & White/gummy & + & + & cocci & - \\
\hline 51 & White/gummy & + & + & rod & - \\
\hline 25 & White/gummy & + & + & $\operatorname{rod}$ & - \\
\hline 21 & Colorless & + & + & $\operatorname{rod}$ & - \\
\hline 55 & White/gummy & - & - & rod & - \\
\hline 99 & Pink/gummy & + & + & rod & - \\
\hline 11 & Colorless & - & - & rod & - \\
\hline 13 & Pink & + & - & $\operatorname{rod}$ & - \\
\hline 28 & Colorless & - & - & cocci & - \\
\hline 202 & White/gummy & + & - & cocci & - \\
\hline 196 & Colorless & - & - & $\operatorname{rod}$ & - \\
\hline
\end{tabular}




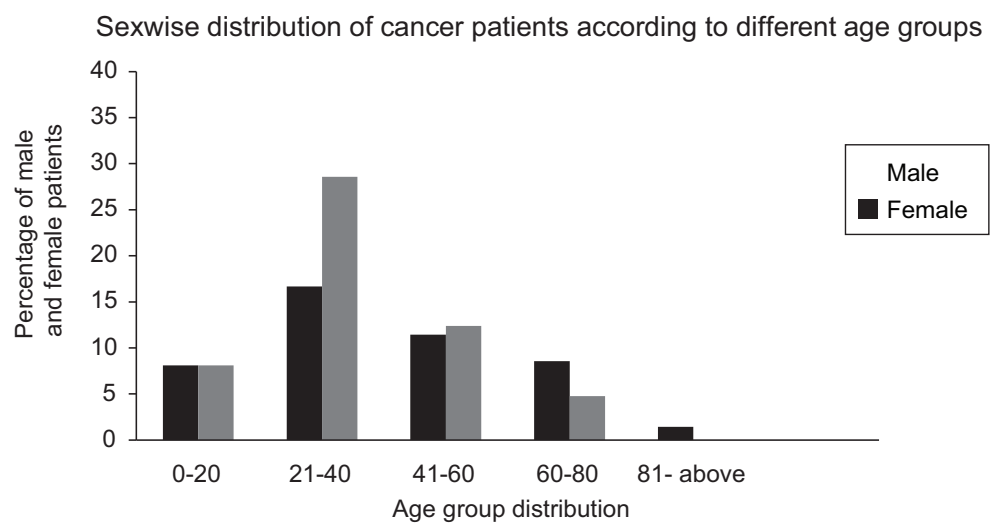

Figure 1. Percentage of female and male cancer patients in different age groupsinvestigated

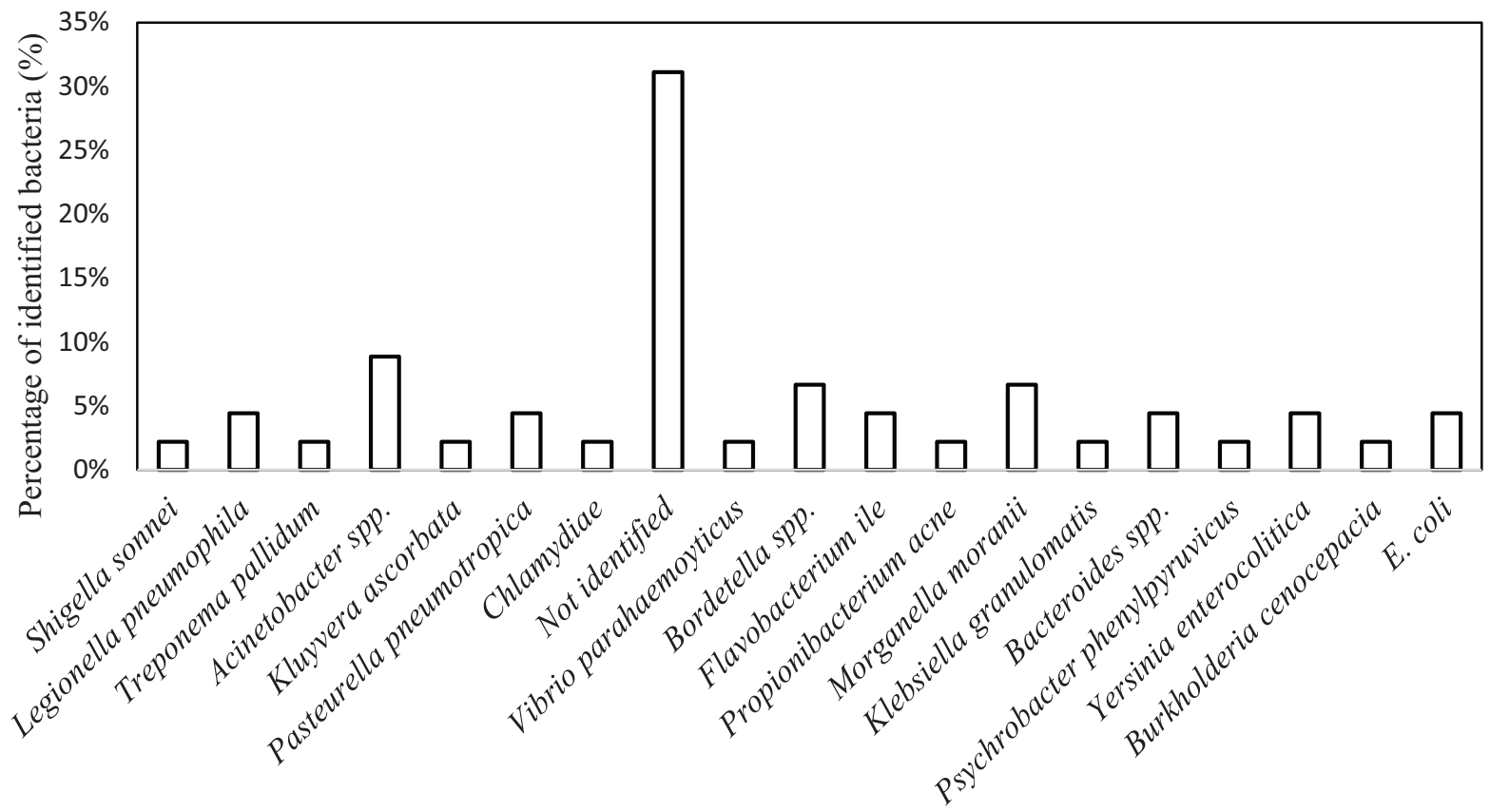

Bacterial identification

Figure 2. Relative percentages ofbacteria present in isolated fluids of cancer patients. Isolate identification was carried out by Gideon (http://web.gideononline.com) by using the results from the biochemical tests carried out as inputs.
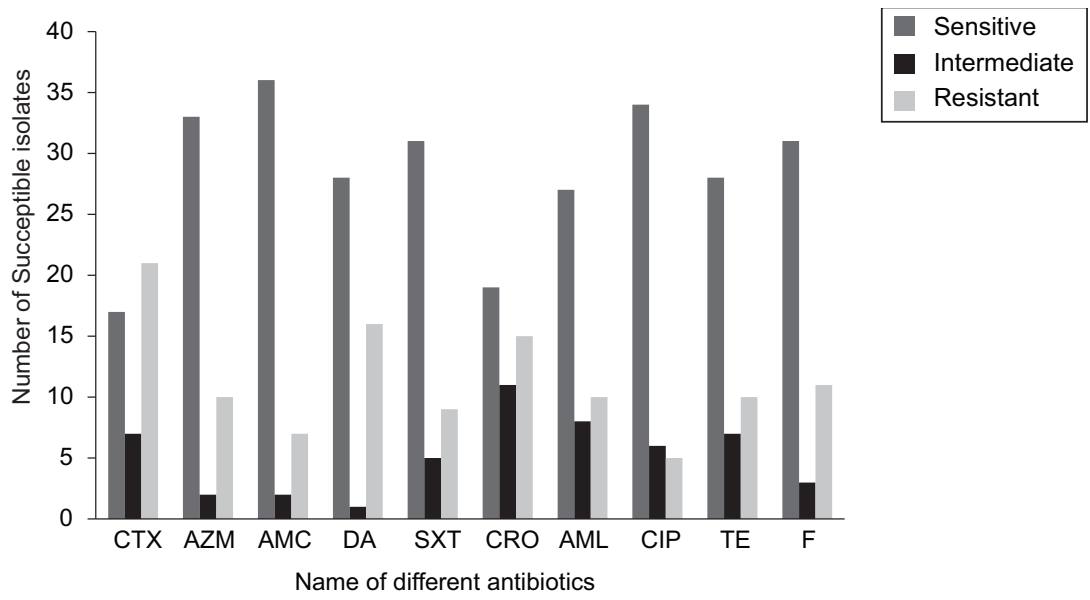

Figure 3. Antibiotic Susceptibility pattern of the isolates (CTX-Cefotaxime, AZM-Azithromycin, AMC-Amoxycillin, DA-Clindamycin, SXT - Sulfamethoxazole, CRO-Ceftriaxone, AML - Amoxyclav, CIP - Ciprofloxacin, TE - Tetracycline, and F-Nitrofurantoin) 


\section{Discussion}

Recently, the emergence of antimicrobial resistance has become a health problem worldwide. Infections due to multidrug-resistant (MDR) Gram-negative bacteria are of special concern, since the therapeutic options are often very limited. Several studies have shown that cancer patients infected with resistant bacteria more often receive inadequate initial empirical antibiotic therapy, which may impair outcomes, increase mortality and prolong hospitalization.

In our study out of 210 samples collected from National Cancer Hospital in Dhaka, Bangladesh, Acinetobacter spp. led the list of pathogens $(8.89 \%)$ followed by Bordetella spp. $(6.67 \%)$, Morganellamoranii(6.67\%), Legionellapneumophila(4.4\%), Escherichia coli (4.4\%), Yersinia enterocolitica(4.4\%), Bacteroides spp. (4.4\%), Shigellasonnei(2.2\%) and Klebsiella granulomatis(2.2\%).Zinner reported that gram-negative bacteraemia remains an important cause of morbidity and mortality in neutropenic patients ${ }^{8}$. In his study, E. coli led the list of pathogens. In our study, $4.4 \%$ of the total gram-negative isolated bacteria were $E$. coli followed by Yersinia enterocolitica (4.4\%) which is similar to a study by Saghir, $2009^{10}$. Previous studies have shown the presence of some multi drug resistant bacterial species among cancer patients. Other Enterobacteriaceae (Citrobacter spp., Enterobacter spp., Proteus spp. andSerratia spp.) are less common, although their frequency may vary according to institution ${ }^{11}$. Significantly, antimicrobial resistance among Enterobacteriaceae has recently been described in cancer and HSCT patients, mainly due to ESBL-producing and Amp-C cephalosporinase hyper producing strains ${ }^{12}$.In another study on 328 bloodstream infections in the pediatric oncology unit at the National Cancer Institute Pseudomonas species, Acinetobacter species, Enterobacter species, Klebsiella species and $E$. coliwere isolated with the following percentages $5.5 \%, 6.7 \%, 2.7 \%, 1.5 \%$ and $2.1 \% 12$. In another study on urinary tract infection in 4 intensive care units in Egypt Klebsiella pneumoniae, Escherichia coli, and Pseudomonas species were the most commonly isolated bacteria ${ }^{14}$.

In vitro activity of different antibiotics was evaluated in our study, most of the isolates were resistant to Cefotaxime, Nitrifurantoin,Clindamycin, Ceftriaxone, and Amoxycillin. Most of the isolates were sensitive to Ciprofloxacin. In an earlier study, it was shown that increasingconsumption of Ceftazidime was associated with decreasing susceptibility of Acinetobacter species and $S$. maltophilia ${ }^{9}$. High resistance rates against Cephalosporins in Ps. aeruginosaand Enterobacteriaceae ${ }^{15}$. In their study, Enterobacter species exhibited $95.8 \%$ resistance to both antibiotics whereas Pseudomonas species exhibited $87.6 \%$ and $66.3 \%$ resistance to Cefotaxime andCeftazidime, respectively.Resistance was high in Escherichia coli and Klebsiella species, which was consistent with a study from Egypt that reported high resistance levels to Cefotaxime (74.4\%) in Gram-negative rods ${ }^{14}$. This high resistance in
Enterobacteriaceaemay be attributed to ${ }^{2}$-lactamase activity ${ }^{16}$.

Although the prevalence of ${ }^{2}$-lactamase producers in our study was low (\%) continued surveillance to confirm the presence of $\mathrm{ES}^{2} \mathrm{~L}$ enzymes among futurecancer isolates may actually reveal a higher prevalence of the enzyme. This is an important future avenue speciallywhen the rates of extended-spectrum ${ }^{2}$-lactamase (ESBL) producing isolates among E. coli and Klebsiella species are shown to be increasing in another study ${ }^{17}$. Studies on the resistance to ß-lactam antimicrobial agents, especially extendedspectrum Cephalosporins and other antimicrobial agents among clinical isolates of Gram-negative bacteria are on the rise worldwide $^{18}$.In Egypt it was reported that extended spectrum ${ }^{2}$ lactamase was detected in $78.6 \%$ and $56 \%$ of $E$. coliand $K$. pneumoniaestrains, respectively ${ }^{14}$. In the present study, no extended spectrum ${ }^{2}$-lactamase was detected in the 45 isolates. One isolate of Klebsiella sp. produced ${ }^{2}$-lactamase among the 149 isolates studied.

Multidrug-resistance organisms (MDRO) such as P. aeruginosa, K. pneumoniae and the other Enterobacteriaceae species with emerging resistance, are important causes of morbidity and mortality in hospitalized critically ill patients and patients with underlying medical condition such as neutropenia and immunosuppressants ${ }^{19}$. The return to the pre-antibiotic era has become a reality in many parts of the world. MDR microorganisms were recently named as the 'ESKAPE' pathogens (Enterococcus faecium, Staphylococcus aureus, Klebsiella pneumoniae, Acinetobacter baumannii, Pseudomonas aeruginosa and Enterobacter species), indicating their 'escape' from the effects of antibacterial agents or the non-existence of newer active antibiotics ${ }^{20}$. In our study, MDR organisms were observed which is alarming. The lack of alternative agents that are active against gram-negative bacteria necessitates the use of measure for controlling emergence of resistance in bacterial strains particularly in cancer patients.

Multidrug resistance is a worldwide problem that does not obey international borders and can indiscriminately affect members of all socioeconomic classes. In this study, resistance to selected antibiotics was studied in 45 clinical samples isolated from a total of 149 samples. It was found that the test isolates were resistant to all antibiotics in variable extents. Extended Spectrum ${ }^{2}$-lactamase activity was absent among the isolates. Only one isolate presented as ${ }^{2}$-lactamase producer.The antibiotics active against MDR bacteria have several disadvantages, including limited clinical experience, a higher incidence ofadverse effects and less knowledge of the pharmacokinetics of the drug. Therefore, a thorough acquaintance with the main characteristics of these drugs is essential in order to safely treat cancer patients with MDR bacterial infections. It can be concluded that, treatment of infections due to MDR bacteria has become a clinical challenge. The presence of MDR bacteria in cancer patients is of particular concern because these patients are immunocompromised. Implementation of antibiotic stewardship 
programs and infection control measures is essential in order to avoid antimicrobial resistance development and dissemination.

\section{References}

1. Genevieve Housman, Shannon Byler, Sarah Heerboth, Karolina Lapinska, Mckenna Longacre, Nicole Snyder, and Sibaji Sarkar. Drug Resistance in Cancer: An Overview. Cancers (Basel). 2014; 6(3): 1769-1792.

2. Lakshmaiah KC, Sirsath NT, Subramanyam JR, Govind BK, Lokanatha D, Shenoy AM.Aspiration in head and neck cancer patients: a single centre experience of clinical profile, bacterial isolates and antibiotic sensitivity pattern.Indian J Otolaryngol Head Neck Surg. 2013 Jul; 65(Suppl 1):144149. doi: 10.1007/s12070-013-0645-7. Epub 2013 Mar 20.

3. Nature Biotechnology. Vol. 18 Supplement. 2000.http://biotech. nature.com

4. C. Lee Ventola. 2015. The Antibiotic Resistance Crisis. Part 1: Causes and Threats.PT. 40(4): 277-283.

5. Carlota Gudiol and Jordi Carratala. 2014. Antibiotic resistance in cancer patients Expert Rev. Anti Infect. Ther.Early online: 1-14.

6. http://web.gideononline.com

7. Bauer AW, Kirby WMM, Sherris JC, et al. 1966. Antibiotic susceptibility testing by a standardized single disk method. Am. J. Clin. Pathol. 45: 493-496.

8. Jarlier V, Nicolas MH, Fournier G, Philippon A. 1988. Extended broadspectrumbeta-lactamases conferring transferable resistance to newer betalactamagents in Enterobacteriaceae: hospital prevalence and susceptibilitypatterns. Rev. Infect. Dis. 10: 867-878.

9. Zinner H S (2000) New pathogens in neutropenic patients with cancer: an update for the new millennium. Int J Antimicrob Agents 16(2): 97101

10. Saghir S, Faiz M, Saleem M, Younus A, Aziz H (2009) Characterization and anti - microbial susceptibility of gramnegative bacteria isolated from bloodstream infections of cancer patients on chemotherapy in Pakistan. Indian J Med Microbiol 27: 341-347.
11. Yadegarynia D, Tarrand J, Raad I, Rolston K. Current spectrum of bacterial infections in patients with cancer. Clin Infect Dis 2003; 37:1144-5

12. Montassier E, Batard E, Gastinne T, et al. Recent changes in bacteremia in patients with cancer: a systematic review of epidemiology and antibiotic resistance. Eur J ClinMicrobiol Infect Dis 2013; 32:841-50.

13. El-Mahallawy H, Sidhom I, Ali El-Din N H, Zamzam M., El-Lamie M M (2005) Clinical and microbiologic determinants of serious bloodstream infections in Egyptian pediatric cancer patients: a one-year study. Int J Infect Dis 9: 43-51.

14. Talaat M, Hafez S, Saied T, Elfeky R, El-Shoubary W, et al. (2010) Surveillance of catheter-associated urinary tract infection in 4 intensive care units at Alexandria university hospitals in Egypt. Am J Infect Control 38: $222-228$.

15. O'Neill E, Humphreys H, Phillips J, Smyth EG. (2006) Thirdgeneration cephalosporin resistance among gram-negative bacilli causing meningitis in neurosurgical patients: significant challenges in ensuring effective antibiotic therapy. J AntimicrobChemother 57: 356-359.

16. Wenzel PR, Sahm FD, Thornsberry C, Draghi DC, Jones EM, et al. (2003) In vitro Susceptibilities of gram-negative bacteria isolated from hospitalized patients in four European countries, Canada, and the United States in 2000-2001 to expanded-spectrum cephalosporins and comparator antimicrobials: implications for therapy. Antimicrob Agents Chemother 47: 3089-3098.

17. Rolston V I (2005) Challenges in the Treatment of Infections Caused by gram-Positive and gram-Negative Bacteria in Patients with Cancer and Neutropenia. ClinInfec Dis 40(S4): S246-S252.

18. Pfaller MA, Jones RN (2000) MYSTIC (Meropenem Yearly Susceptibility Test Information Collection) results from the Americas: Resistance implications in the treatment of serious infections. J Antimicrob Chemother 46: 25-37.

19. Mallik K, Carmelita E, Craig A, Susan F, Kenneth V (2005) Bacterial infections in low-Risk, Febrile Neutropenic Patients. Cancer 104: 422-426.

20. Giamarellou H (2010) Multidrug-resistant gram-negative bacteria: how to treat and for how long. Int J Antimicrob Agents 36(S2): S50-S54. 